\title{
Molecular Community Analysis of Arbuscular Mycorrhizal Fungi in Roots of Geothermal Soils in Yellowstone National Park (USA)
}

\author{
Susann Appoloni • Ylva Lekberg • Michael T. Tercek • \\ Catherine A. Zabinski • Dirk Redecker
}

Received: 25 February 2008 / Accepted: 4 March 2008/Published online: 1 May 2008

(C) Springer Science + Business Media, LLC 2008

\begin{abstract}
To better understand adaptation of plants and their mycorrhizae to extreme environmental conditions, we analyzed the composition of communities of arbuscular mycorrhizal fungi (AMF) in roots from geothermal sites in Yellowstone National Park (YNP), USA. Arbuscular mycorrhizal fungi were identified using molecular methods including seven specific primer pairs for regions of the ribosomal DNA that amplify different subgroups of AMF. Roots of Dichanthelium lanuginosum, a grass only occur-
\end{abstract}

Electronic supplementary material The online version of this article (doi:10.1007/s00248-008-9384-9) contains supplementary material, which is available to authorized users.

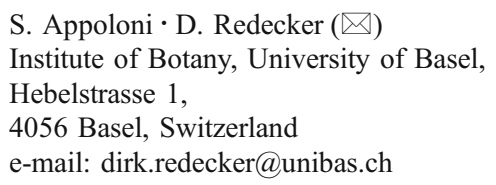

ring in geothermal areas, were sampled along with thermal and nonthermal Agrostis scabra and control plants growing outside the thermally influenced sites. In addition, root samples of Agrostis stolonifera from geothermal areas of Iceland were analyzed to identify possible common mycosymbionts between these geographically isolated locations. In YNP, 16 ribosomal DNA phylotypes belonging to the genera Archaeospora, Glomus, Paraglomus, Scutellospora, and Acaulospora were detected. Eight of these phylotypes could be assigned to known morphospecies, two others have been reported previously in molecular studies from different environments, and six were new to science. The most diverse and abundant lineage was Glomus group A, with the most frequent phylotype corresponding to Glomus intraradices. Five of the seven phylotypes detected in a preliminary sampling in a geothermal area in Iceland were also found in YNP. Nonthermal vegetation was dominated by a high diversity of Glomus group A phylotypes while nonthermal plants were not. Using multivariate analyses, a subset of three phylotypes were determined to be associated with geothermal conditions in the field sites analyzed. In conclusion, AMF communities in geothermal soils are distinct in their composition, including both unique phylotypes and generalist fungi that occur across a broad range of environmental conditions.

\section{Introduction}

Volcanic activity in Yellowstone National Park (YNP) in the Northwestern USA has caused the formation of geysers, thermal springs, mud pots, and steam vents (fumaroles). Microbes from aquatic geothermal environments have been characterized extensively, including cyanobacteria, green sulfur bacteria, and archaea (e.g., [20]). Soils that occur in 
these geothermally active areas often show an elevated temperature and altered chemistry, with elevated levels of manganese, iron, aluminum, arsenic, and sulfide [14, 32]. Plant growth is often sparse in these areas and restricted to a few species. The heat-resistant grass Dichanthelium lanuginosum (Schmoll) Spellenb. (Dichanthelium acuminatum (Sw.) Gould and Clark; hot springs panicgrass, Poaceae) occurs exclusively in geothermal areas within YNP and can grow in soil temperatures reaching $57^{\circ} \mathrm{C}$ [38]. A second grass, Agrostis scabra Willd., (rough bentgrass, Poaceae) occurs in geothermal as well as nonthermal sites. It has been suggested that thermal and nonthermal A. scabra are reproductively isolated and represent different species [42].

Several studies have addressed possible mechanisms that enable plants to survive in geothermal areas, including altered root morphology, expression of heat-shock proteins [37], and use of water and steam resources to ameliorate heat stress [11]. Furthermore, growth within moss mats has been observed in some geothermal sites where the moss may ameliorate harsh environmental conditions for plants at some stages of their life cycle [43].

Besides the plants' tolerance and avoidance strategies, interactions with symbiotic fungi may confer increased fitness in geothermal environments. Fungal endophytes from the ascomycete genus Curvularia infected with a mycovirus confer significantly higher temperature tolerance to Dichanthelium in YNP $[22,30]$ by an unknown mechanism. Plants in geothermal areas are also consistently colonized by arbuscular mycorrhizal fungi (AMF), opening the possibility that this symbiosis may play a role in plant survival under these extreme conditions [3].

Arbuscular mycorrhiza is a mutualistic symbiosis formed between the majority of land plants and fungi from the phylum Glomeromycota. Many plants depend to a large extent on this interaction for their mineral nutrition. The host plants provide carbohydrates to the fungi, while the fungi increase the uptake of phosphorus and other nutrients of poor mobility [8] and improve resistance against certain pathogens [2]. The mycorrhizal symbiosis is of particular importance under nutrient-limiting conditions [35]. Fungal morphospecies and isolates may differ considerably in their ability to alleviate stress factors, such as high $\mathrm{Al}^{3+}$ concentrations and low $\mathrm{pH}$ [4]. If environmental conditions favor certain symbiont combinations, then specialized AMF communities would be expected. The distinct chemical and physical conditions in geothermal areas [3] may result in unique AMF communities. One objective of this research was to characterize AMF community composition in geothermal areas within YNP.

AMF have traditionally been classified and identified based on microscopic characteristics of spores. Currently, approximately 200 morphospecies are distinguished (http://www.amf-phylogeny.com). The task of identifying spores based on their morphology is notoriously difficult, and similar spore types can be formed by fungi that are distantly related [29]. In addition, spore formation is not always correlated with the extent of root colonization, and thus, evaluations of AMF communities based on spores alone may not accurately reflect the AMF community that is interacting with plants. To address this potential problem, molecular techniques that allow identification of AMF within roots were developed during the last decade, targeting the nuclear-encoded ribosomal genes [27]. Field studies based on molecular markers have provided numerous new insights into the dynamics of AMF communities and have presented evidence for host preference and host specificity [13], effects of season and plant host development stage [16], as well as an influence of agriculture and ecosystem type [15]. These studies have also shown that while some AMF species appear to be rather limited in their distribution, others are generalists and surprisingly widespread [25].

In this study, we used molecular methods to analyze AMF communities in geothermal areas in YNP to study the influence of extreme environmental factors on these communities. The steep edaphic gradients in geothermal areas of YNP provided an intriguing environment to study the response of AMF communities to environmental factors. To exclude possible geographic effects, we also sampled and analyzed a geothermal area in Iceland.

As nothing was previously known about AMF species occurring under these conditions, we expected two alternative scenarios: (1) unique AMF phylotypes specialized for geothermal conditions and not occurring in other environments; (2) AMF phylotypes with wide tolerances also occurring in other environments.

\section{Methods}

Description of Geothermal Sites, Host Plants, and Sampling Procedures

We sampled from three geothermal areas and their surrounding transition zones in YNP: Rabbit Creek (RC)

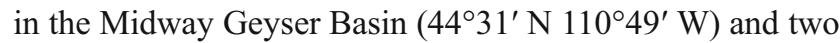
geothermal areas located $50 \mathrm{~m}$ apart in the Upper Geyser Basin in the Lone Star Geyser Area (LG 1 and 2, respectively, $\left.44^{\circ} 34^{\prime} \mathrm{N} 110^{\circ} 48^{\prime} \mathrm{W}\right)$. For comparison, eight glomeromycotan rDNA sequences from $D$. lanuginosum roots and spores from a third site in the Lone Star Geyser Basin (LG3, Lekberg et al. unpublished; $44^{\circ} 26^{\prime} \mathrm{N} 110^{\circ} 49^{\prime}$ $\mathrm{W})$ were included in the phylogenetic trees. These 
sequences were obtained using the same molecular methods as the ones described here.

At each site, D. lanuginosum was growing in the warmest areas with thermal $A$. scabra. The nonthermal $A$. scabra was found in geothermally influenced, but lowertemperature soils ("transition zone") together with other nonthermal plant species. The geothermal environment and transition zone at RC were previously characterized by Bunn and Zabinski [3] where soil temperatures of $31-42^{\circ} \mathrm{C}$ and soil $\mathrm{pH}$ values of 3.4-4.8 were reported. Furthermore, chemical profiles of geothermal and adjacent transition area soils were analyzed and appeared to differ significantly from each other. Iron was four times higher in geothermal soils compared to transition area soils, whereas $\mathrm{Zn}$ and $\mathrm{Mn}$ were much lower. Dichanthelium plants growing in the geothermal area were heavily colonized (averaging 53\% colonized root length) throughout the summer, suggesting that this was an appropriate site for our study.

Field sites at LG1 and LG2 are located approximately $11 \mathrm{~km}$ from RC. Plants at LG1 grow on the moss Racomitrium canescens, but this is lacking at LG2. Both soils are acidic with $\mathrm{pH}$ around 4 at LG1 and 4.8 at LG2.

Sample collection is summarized in Supp. Table 1. At all sampling dates, the temperatures at a depth of $2 \mathrm{~cm}$ in thermal areas were substantially higher (averages of $34.9^{\circ}$ $\mathrm{C}, 45^{\circ} \mathrm{C}, 41.8^{\circ} \mathrm{C}, 41.9^{\circ} \mathrm{C}$ ) than in transition areas in $\mathrm{RC}$ (averages $25^{\circ} \mathrm{C}, 23.9^{\circ} \mathrm{C}$ ) or nonthermal control plants in LG1 and LG2 $\left(25^{\circ} \mathrm{C}\right.$ and $\left.27^{\circ} \mathrm{C}\right)$.

We collected samples in September 2003, October 2004, and June 2005 from RC, and in May 2005 from LG1 and LG2.

Whole plants were excavated, roots were stored at $4{ }^{\circ} \mathrm{C}$, washed in the laboratory at Montana State University within $24 \mathrm{~h}$, and divided into aliquots of approximately $100 \mathrm{mg}$. After transport to Switzerland, they were frozen in liquid nitrogen and stored at $-80^{\circ} \mathrm{C}$, or they were lyophilized before transport.

In addition to the collections in geothermal and transition zones, we also collected mixed-species root cores (depth: approximately $5 \mathrm{~cm}$ ) from nonthermal adjacent areas in LG1 and LG2, which contained A. scabra and other plant species that were not determined ("control plants").

In August 2005, roots of Agrostis stolonifera (creeping bentgrass, Poaceae) growing in a geothermal site at Ölkelduhals (Iceland) were sampled. Soil pH was 4-4.5 and soil temperature ranged from $37-41^{\circ} \mathrm{C}$ in thermal soils. The plants that were sampled in Iceland were washed and frozen at $-80^{\circ} \mathrm{C}$ within 3 days.

A total of 37 root samples from 33 plants from YNP, and three plants from Iceland were analyzed. Internal root colonization was estimated qualitatively after staining subsamples of the roots with trypan blue according to standard procedures [19].
Molecular Identification

\section{DNA Extraction}

Aliquots of $100 \mathrm{mg}$ roots were ground in liquid nitrogen with mortar and pestle or with a pellet pestle within the 1.5$\mathrm{ml}$ tube. DNA was extracted from roots with a DNeasy Plant Mini Kit (Qiagen, Hilden, Germany) according to the manufacturer's instructions. DNA was eluted in one step in $30 \mu$ elution buffer. Purified DNA was diluted 1:10 and 1:100 in Tris ethylene diamine tetraacetic acid (TE) buffer and used as template for the first polymerase chain reaction (PCR) of the nested procedure.

\section{Polymerase Chain Reaction}

PCR was performed in a nested procedure [15] using Taq polymerase from Amersham (Basel, Switzerland), $2 \mathrm{mM}$ $\mathrm{MgCl}_{2} 0.5 \mu \mathrm{m}$ primers, and $0.25 \mathrm{mM}$ of each desoxynucleotide phosphate. The first round of amplification was performed using universal eukaryote primers NS5 and ITS4 [46]. The cycling parameters were: $3 \mathrm{~min}$ at $95^{\circ} \mathrm{C}$, followed by 30 cycles of $30 \mathrm{~s}$ at $95^{\circ} \mathrm{C}, 30 \mathrm{~s}$ of $51^{\circ} \mathrm{C}$, and $2 \mathrm{~min}$ at $72^{\circ} \mathrm{C}$. The program was concluded by a final extension phase of $10 \mathrm{~min}$ at $72^{\circ} \mathrm{C}$. PCR products were diluted 1:100 in TE buffer and used as template in the second round. Eight separate PCR reactions were performed using the following primer pairs [15, 40]: GLOM1310/ITS4i, ARCH1311AB/ITS4i, PARA1313/ITS4i, ACAU1661/ITS4i, NS5/GLOM5.8R, GIGA1313/GIGA5.8R, GLOM1310/GLOM5.8R, and LETC1677/ITS4i.

Primer sequences and target AMF clades were: ACAU1661 (TGA GAC TCT CGG ATC GGG, Acaulosporaceae), ARCH1311AB (equimolar mixture of TGC TAA ATA GCT AGG CTG C and TGC TAA ATA GCC AGG CTG T; Archaeospora or Paraglomus), GIGA1313 (CTA AAT AGT CAG GCT AWT CTT, Gigasporaceae), GLOM1310 (AGC TAG GYC TAA CAT TGT TA, Glomus group A), LETC1677 (CGG TGA GTA GCA ATA TTC G, Glomus group B), PARA1313 (CTA AAT AGC CAG GCT GTT CTC, Paraglomus), GIGA5.8R (ACT GAC CCT CAA GCA KGT, Gigasporaceae), GLOM5.8R (TCC GTT GTT GAA AGT GAT, Glomus group A), ITS4i (TTG ATA TGC TTA AGT TCA GCG).

The PCR parameters for the second round were: $3 \mathrm{~min}$ at $95^{\circ} \mathrm{C}$, followed by 30 cycles of $45 \mathrm{~s}$ at $95^{\circ} \mathrm{C}, 50 \mathrm{~s}$ of $61^{\circ} \mathrm{C}$, and $1.5 \mathrm{~min}$ at $72^{\circ} \mathrm{C}$. The program was concluded by a final extension phase of $10 \mathrm{~min}$ at $72^{\circ} \mathrm{C}$. A "hot start" at $61^{\circ} \mathrm{C}$ was performed manually to prevent nonspecific amplification. In order to check the success of amplification, PCR products from the second round of nested PCR were run on agarose gels (2:1\% NuSieve/ 
SeaKem, FMC, Rockland, ME, USA) in Tris or Acetate buffer at $90 \mathrm{~V}$ for $45 \mathrm{~min}$.

\section{Cloning and Sequencing}

PCR products were cloned into a pGEM-T vector (Promega/Catalys, Wallisellen, Switzerland). Inserts from ten clones per ligation were reamplified, digested with HinfI and $M b o \mathrm{I}$, and run on agarose gels. These enzymes have recognition sequences of four nucleotides and have proven to be useful in the gene region of the Glomeromycota used here [40]. Restriction fragment patterns were compared to a database based on Good-Enough RFLP Matcher [7]. Representatives of restriction types newly found in the respective sample were, then, sequenced in both directions. PCR products to be sequenced were purified using a High Pure Kit from Hoffman LaRoche (Basel, Switzerland). A BigDye Terminator Cycle Sequencing Kit (Applied Biosystems, Foster City, CA, USA) was used for labeling. Samples were run on an ABI 310 capillary sequencer. Sequences were deposited in the European Molecular Biology Laboratory database under the accession numbers AM942452 to AM942537 and AM905249 to AM905256.

\section{Sequence Analysis}

Sequences were aligned to previously published sequences in PAUP*4b10 [39]. The glomeromycotan origin of the sequences was verified by Basic Local Alignment and Search Tool [1] and by phylogenetic analysis of an alignment of 5.8S subunit sequences [28]. Separate ITS alignments were used for Archaeospora, Paraglomus, Acaulospora and Glomus group A [34]. In addition, an alignment of the partial $3^{\prime}$ end of the 18S rDNA small subunit was compiled for the $18 \mathrm{~S}$ sequences amplified with GIGA5.8R, GLOM5.8R, GLOM1310, PARA1313, and ARCH1311AB. Representative sequences of each sequence type were checked manually for possible chimaeras, which were excluded from the analysis.

Phylogenetic trees were primarily obtained by distance analysis using the neighbor joining algorithm in PAUP* using the Kimura two-parameter model and a gamma shape parameter $=0.5$. Neighbor-joining bootstrap analyses were performed with 1,000 replications. Results were verified by performing maximum likelihood analyses based on parameters estimated in Modeltest 3.5 [26].

\section{Definition of Sequence Phylotypes}

Single morphospecies and even spores of Glomeromycota contain multiple minor variants of rDNA sequences. It is not possible to assign a single sequence to a certain species or a fungal isolate. Sequence phylotypes were, therefore, defined in a conservative manner as consistently separated monophyletic groups in the phylogenetic trees. Only those clades were used that were supported by neighbor joining bootstrap analyses and also present in the respective maximum likelihood tree. The sequence phylotypes were designated after the major AM fungal clade in which they were placed, followed by a numerical index ( $\mathrm{x}$ in the following examples) identifying the type: Glomus group A (GLOM-Ax, [34]), Glomus group B (GLOM-Bx), Acaulosporaceae (ACAU-x), Gigasporaceae (GIGA-x), Paraglomus (PARA-x), Archaeospora sensu lato (ARCH-x [24]). Numbering is consistent with prior studies conducted in the laboratory in Basel (e.g., [15]).

\section{Data Analyses}

The presence or absence of each AMF phylotype in each root sample was used to construct a sampling effort curve in EstimateS [6]. In order to investigate the influence of environmental factors (site, temperature, and host plant) on the distribution of AMF phylotypes, ordination analyses were conducted in CANOCO for Windows v. 4.5 [41] using the presence or absence data for each root sample. Environmental variables (site, host plant, sampling date) were coded by dummy variables $(1 / 0)$ with the exception of temperature. Initial Detrended Correspondence Analysis suggested a unimodal character of the data response to the sample origin (the lengths of gradients were $>4$ ); therefore, the Canonical Correspondence Analysis (CCA) was used. The analyses were conducted using the following settings: focus on interspecies distances, biplot scaling, no transformation, downweighting of rare species.

\section{Results}

AM Fungal Lineages and Their Frequency

PCR products of glomeromycotan fungi were obtained from 29 out of 36 root samples. With the exception of Glomus group B (primer LETC1677), all glomeromycotan lineages targeted by the primer pairs were detected, including Glomus group A, Acaulosporaceae, Gigasporaceae, Archaeospora, and Paraglomus. Mostly in the absence of the target taxa, some primers amplified sequences of Ascomycota or (to a much smaller extent) Basidiomycota. This was particularly the case with primers ARCH1311AB, PARA1313, and ACAU1661.

Based on phylogenetic analyses of the five different datasets (Figs. 1, 2, and 3, Figs. S1 and S2), 18 phylotypes were defined, eight of which could be reliably assigned to known morphospecies from other environments. The largest diversity was found in Glomus group A (ten 
phylotypes). Representatives of this group were present in $76 \%$ of all positive samples. Three phylotypes from Glomus group A could be assigned to known species: Glomus intraradices (GLOM-A1), Glomus aureum (GLOM-A11) and Glomus diaphanum (GLOM-A13). Because only ITS sequences were available from $G$. diaphanum, this relationship could only be demonstrated using the Glomus group A ITS dataset (Fig. S1). Phylogenetic analysis of 5.8S subunit/ITS2, as well as characteristic sequence motifs in the ITS1 region (not shown), were used to confirm some clades receiving only weak support by analysis of the $18 \mathrm{~S}$ subunit (e.g., GLOM-A1, GLOM-A7 a, b, c in Fig. 1).

One of the two phylotypes from the Acaulosporaceae, ACAU-6, was found frequently and showed a close phylogenetic relationship to Acaulospora morrowiae, whereas ACAU-7 was only detected once (Fig. S2). The Archaeospora phylotype ARCH-5 was found consistently in geothermal environments in YNP. This phylotype belongs to a major clade within Archaeospora as defined by Morton and Redecker [24] that is only known from environmental sequences. It is very distantly related to known morphospecies (Fig. 2). In a recent revision, the genus Archaeospora was divided into the two genera Ambispora and Archaeospora [36, 45]. According to this nomenclature, the clade containing ARCH-5 would constitute an unknown glomeromycotan genus.

Three phylotypes of Paraglomus were distinguished (Fig. 3), two of which fell within the morphospecies Paraglomus occultum (PARA-2, PARA-3). The third (PARA-1) corresponds to the newly described species Paraglomus laccatum [31] and has been previously detected in a number of environments (e.g., [15]). Only one phylotype (GIGA-4) of Scutellospora (Gigasporaceae) was found, which was closely related to S. pellucida (not shown). The most frequently found phylotype was GLOMA1 (G. intraradices), found in $41.7 \%$ of the samples, followed by GLOM-A11, GLOM-A26, and PARA-1 (all 19.7\%) and GLOM-A-13, ARCH-5, and ACAU-6 (all $16.7 \%$ ). In all, we found six new phylotypes in YNP that were distinct from known sequences. Whereas some new phylotypes appear to be rare and were only found once (GLOM-A24, ACAU7), others were found more than once (GLOM-A27, GLOM-A7a) and two were detected regularly (ARCH-5 and GLOM-A26).

In the preliminary sampling in Iceland, seven phylotypes were detected, five of which were also found in YNP (GLOM-A1, PARA-1, GLOM-A7a, GLOM-A7b, ACAU6). GLOM-A7a is currently only known from Iceland and YNP, whereas GLOM-A7b has been detected within roots in molecular field studies from other habitats [33, 40].

Root samples harbored between zero and five phylotypes, with many root samples from the hottest soils being apparently nonmycorrhizal. The sampling effort curve (Fig. S3) showed that in the case of Dichanthelium and the Rabbit Creek field site, the major proportion of the phylotype diversity was characterized. In nonthermal $A$. scabra, fewer plants were sampled, but the curve had a similar trajectory as in Dichanthelium. The phylotype richness in thermal A. scabra clearly shows the tendency to stabilize on a much lower level than for $D$. lanuginosum and non-geothermal A. scabra. The sampling in Iceland as well as LG1 and LG2 (not shown) was not exhaustive and provided only a first sample of phylotypes occurring in these sites. However, the purpose of analyzing these samples was to obtain a first insight into the phylotypes occurring across sites, and this analysis indicated that there were a number of phylotypes occurring in more than one site.

AMF Community Structure in Geothermal and Nongeothermal Samples

A group of three phylotypes was consistently and exclusively found in geothermal samples, i.e., in D. lanuginosum and thermal A. scabra but never in nonthermal A. scabra and control plants: ARCH-5, ACAU-6, and PARA-3. Two other phylotypes (ACAU-7 and GIGA-4) were only detected once in thermal samples; therefore, the distribution of these phylotypes cannot be addressed reliably.

Multivariate analyses were conducted on the whole dataset as well as on a subset containing only samples from the more exhaustively sampled Rabbit Creek field site. In the species-environmental variable biplots based on these analyses, the group of three geothermal-associated AMF phylotypes was associated with the temperature gradient and the vectors of the host plants $D$. lanuginosum and thermal A. scabra.

As there was an apparent trend for the absence of AMF colonization in root samples from higher temperatures, a new category "NoSpecies" was created for some analyses to visualize these samples in the biplots. The introduction of this category did not affect the conclusions about the grouping of the three geothermal-associated phylotypes.

With only temperature and host plants included as environmental factors in an analysis of the complete dataset (Fig. 4), three distinct groups of AMF emerged that were associated with A. stolonifera from Iceland, thermal Agrostis or Dichanthelium, and nonthermal A. scabra or control plants, respectively. A similar separation of two groups comprising thermal A. scabra or Dichanthelium and nonthermal A. scabra or control plants was obtained by a parallel analysis of the well-sampled Rabbit Creek site only (Fig. S4).

Interestingly, in the field sites in YNP studied here, Glomus group A phylotypes, did not associate with thermal 


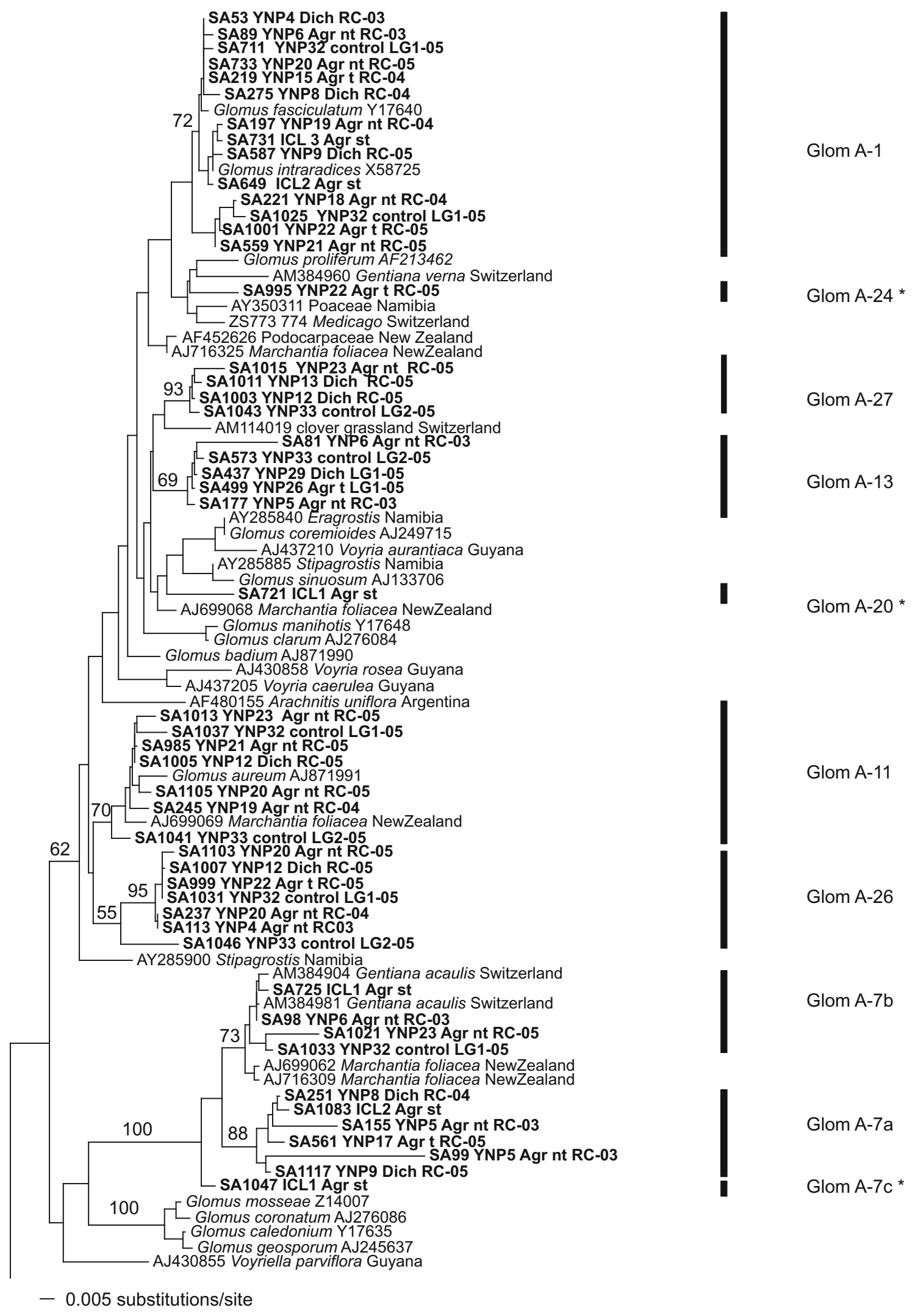


Figure 1 Phylogenetic tree of Glomus group A based on partial 18S rDNA. A tree comprising all glomeromycotan lineages was obtained by neighbor joining, only the Glomus group A clade is shown here for clarity. Numbers above branches denote bootstrap values (percent) from 1,000 replications. Sequences obtained in the present study are shown in boldface. They are labelled with the internal sequence identification number (e.g. SA53), the sample code (YNPX for Yellowstone, ICLx for Iceland, see Table S1), an abbreviation of the host plant (Dich for D. lanuginosum, Agr $t$ for thermal A. scabra, Agr $n t$ for nonthermal A. scabra, control for unidentified nonthermal control plants, and Agr st for A. stolonifera), the location (RC, LG1, LG2, Iceland, see text $)$ and the sampling date $(03=$ Sept $2003,04=$ Oct 2004, 05=May-June 2005). Sequence phylotypes labelled with a star $\left.{ }^{*}\right)$ were only found in one root sample. Sequences from the databases are labelled as follows: accession number, host plant, and country of origin for root-derived sequences; fungal species and accession number for spore-derived sequences
Agrostis or Dichanthelium in the biplots but with nonthermal samples. A striking example were the samples from LG1 and LG2, where only one phylotype from Glomus group A (GLOM-A13) was detected, whereas the control samples taken nearby from nonthermal plants contained a rich variety of phylotypes from this clade. Overall, GLOMA phylotypes were significantly more diverse in nonthermal plants in YNP (average: 2.90 phylotypes per sample) than in thermal plants $(1.13 ; P=0.0002, t$-test $)$. Furthermore, GLOM-A phylotypes were significantly more diverse compared with all other phylotypes in nonthermal plants (0.50; $P<0.0001, t$-test). There was no significant difference in phylotype richness between Glomus group A and other phylotypes in thermal plants.
Figure 2 Phylogenetic tree of Archaeospora based on $5.8 \mathrm{~S}$ rDNA and ITS2. The tree was obtained by neighbor joining. Numbers above branches denote bootstrap values from 1,000 replications. For an explanation of the label details, see Fig. 1. For comparison, some sequences from another study are included, which originate from another field site LG3 in YNP (Lekberg et al. unpublished)

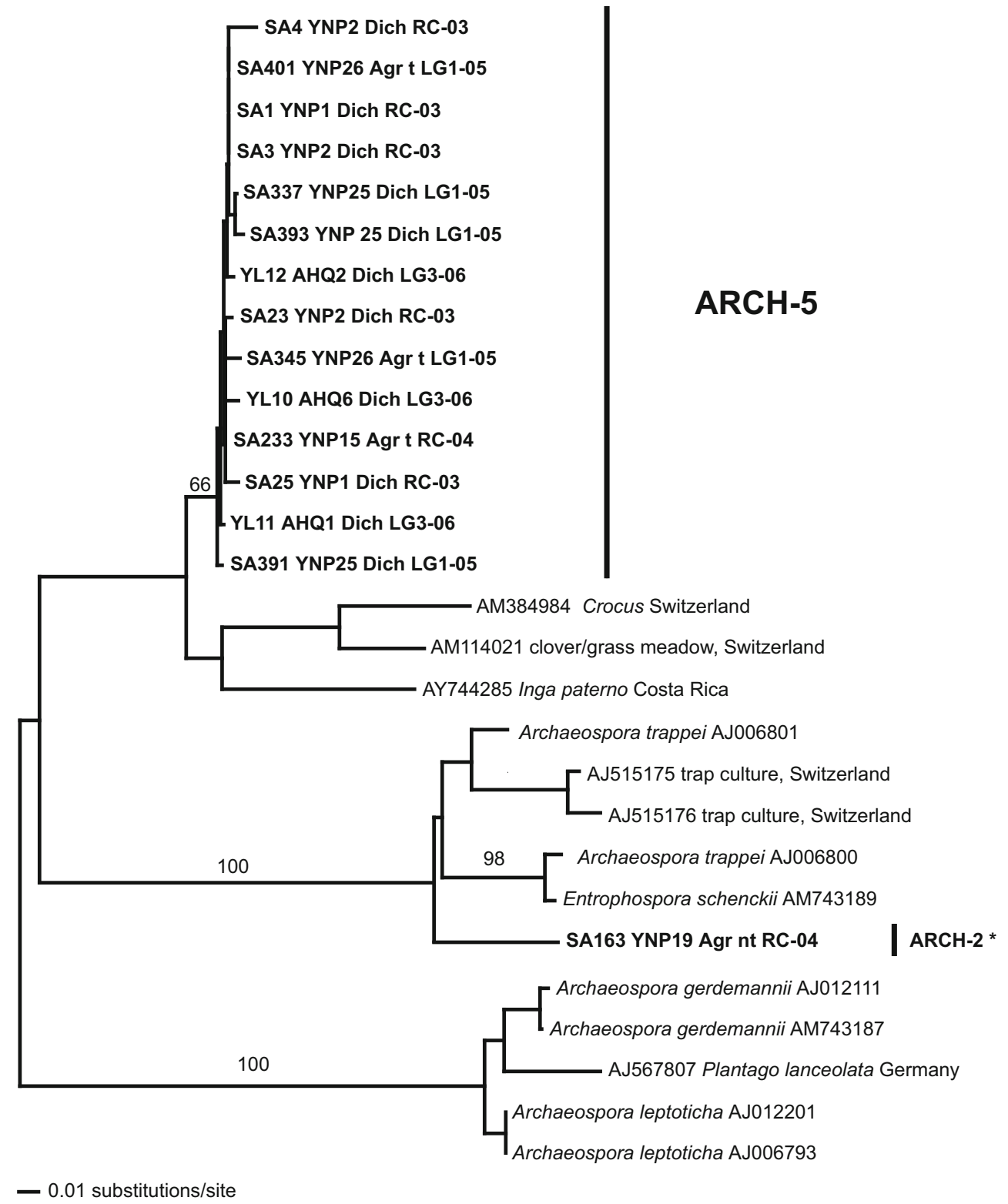


Figure 3 Phylogenetic tree of Paraglomus based on 5.8S rDNA and ITS2. The tree was obtained by neighbor joining. Numbers above branches denote bootstrap values from 1,000 replications. For an explanation of the label details, see Fig. 1. For comparison, some sequences from another study are included, which originate from another field site LG3 in YNP (Lekberg et al. unpublished)

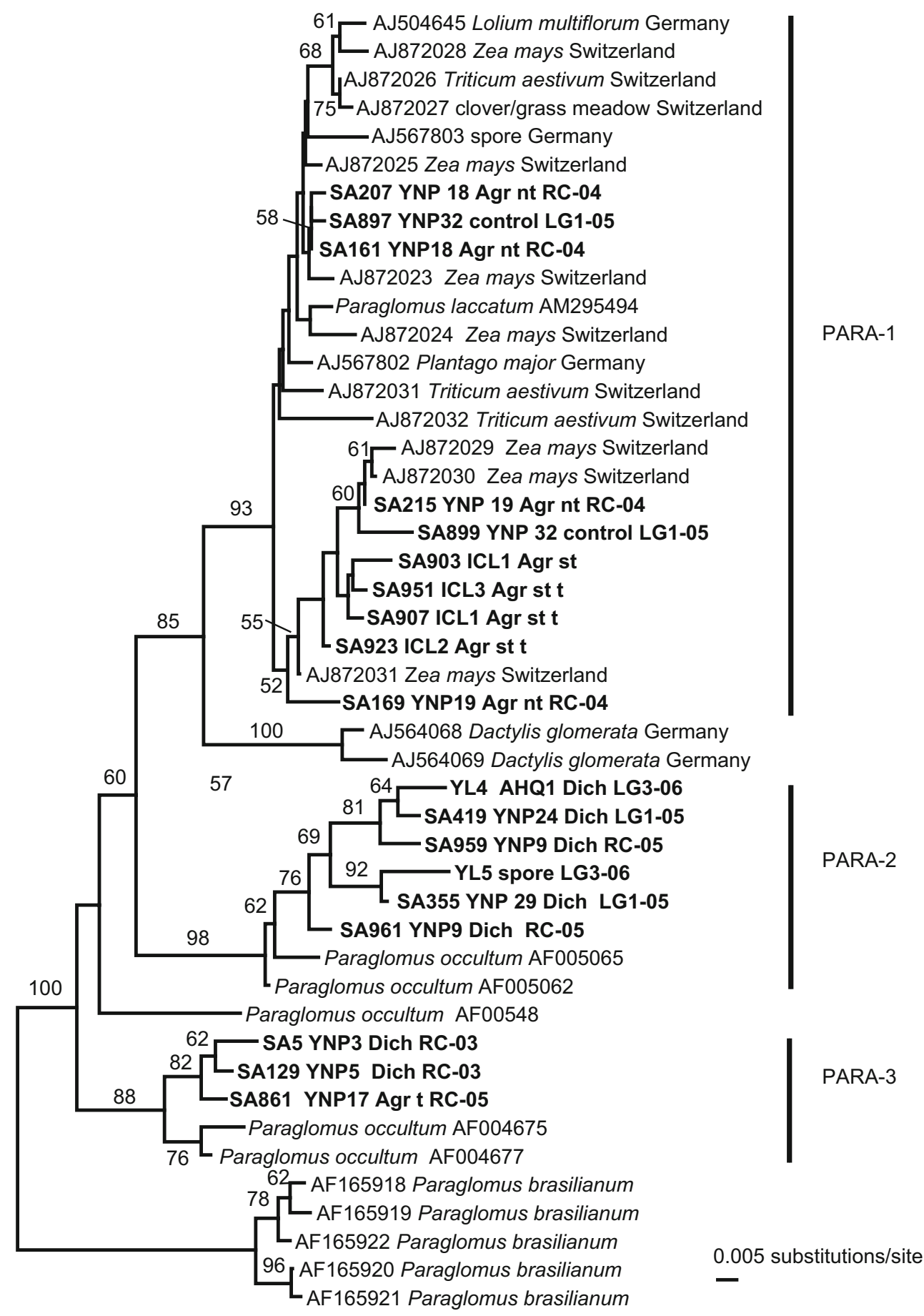

\section{Discussion}

This is the first study using molecular methods to characterize AMF community composition in geothermal soils. Previous studies have addressed effects of soil warming on AMF in greenhouse experiments [12] or root organ cultures [9], but those studies focused on lower soil temperature than was observed here. Tropical soils may also often reach temperatures above $40^{\circ} \mathrm{C}$ [10], but to our knowledge, the effect of this warming on AMF communities has not been addressed. It should be emphasized, however, that in a field study as the one presented here, the temperature factor cannot be separated from the possible influence of the host plants, which occur within specific temperature ranges and the unique soil chemistry of the geothermal soils.

Whether all phylotypes we identified correspond to species and whether biological species concepts can be 


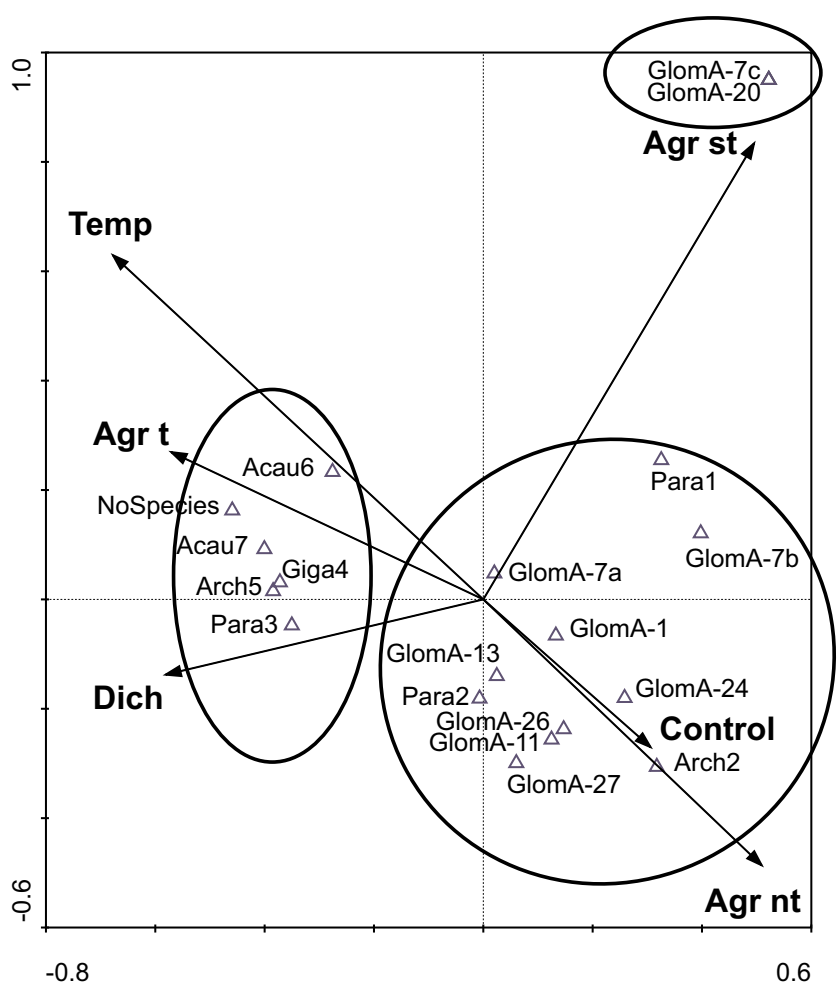

Figure 4 Species-environmental variables biplot $($ arrows $=$ environmental variables, triangles $=$ species $=$ AMF phylotypes) showing a CCA analysis of the complete dataset with environmental variables restricted to temperature and host plants. The $x$-axis represents the first canonical axis, the $y$-axis represents the second canonical axis. Dich $=$ D. lanuginosum, Agr $t=$ thermal A. scabra, Agr $n t=$ nonthermal $A$. scabra, Control $=$ nonthermal control plants, $A g r$ st $=$ A. stolonifera , and Temp $=$ Temperature. This biplot shows the distinct cluster of AMF phylotypes that associates with thermal plant species (Dich and Agr t) and is positively correlated with the temperature gradient. Ellipses were drawn around this cluster and the groups of phylotypes associated with YNP nonthermal samples and A. stolonifera to highlight them

applied in the putatively asexual Glomeromycota remains to be addressed. Nonetheless, we detected a considerable proportion of AMF phylotypes in YNP that have been found elsewhere. In particular, the ubiquitous generalist $G$. intraradices has been found in an astonishingly broad range of environments, including arable soils in Switzerland, Germany [15] and Zimbabwe [21], high montane meadows in the Swiss Alps [40] and in Prunus africana roots from Ethiopia [47]. In fact, the GLOM-A1 phylotypes detected in YNP fall within the range of ITS sequence variation of a single spore of a $G$. intraradices isolate from Switzerland [17]! This range served as a molecular working species definition here (Fig. S1). The fact that this fungus has now been detected in an environment as extreme as thermal soils of YNP has interesting implications for its biology. Koch et al. [18] found that even using highly polymorphic genetic markers, isolates of this species from Switzerland and Canada were surprisingly similar, supporting its status as a widespread generalist.
Multivariate analyses suggested that although generalists such as $G$. intraradices may occur in thermal as well as nonthermal soils, the composition of AMF communities in $D$. lanuginosum and thermal A. scabra in geothermal environments was distinct from A. scabra and control plants in nonthermal environments. Out of the 16 phylotypes detected in YNP, three (ARCH-5, ACAU-6, and PARA-3) showed a clear tendency to occur predominantly at higher soil temperatures and in association with thermal A. scabra and D. lanuginosum.

The phylotype ARCH-5 has not been described outside YNP and belongs to a third major lineage within the genus without any described morphospecies. Members of this clade have been detected in Podocarpaceae roots [33] as a rare phylotype in a clover-grass meadow in Switzerland [15] and abundantly in roots of submerged water plants from oligotrophic lakes (I. Paradi, personal communication). Interestingly, ACAU-6 (A. morrowiae) was found in both YNP and Iceland. This species was previously reported as an efficient plant growth promoter in acidic and high aluminum soils [5], which may explain its occurrence in the hot, acidic soils of geothermal areas. PARA-3 falls within the morphological range of $P$. occultum (together with PARA-2) but can be distinguished genetically and, as demonstrated here, also ecologically as they showed different distribution patterns (Fig. 4). P. occultum was reported to be associated with $G$. diaphanum (GLOM-A13) in acid mine spoils in West Virginia [23], but it is uncertain if that Paraglomus isolate corresponded to either PARA-2 or PARA-3 found here. In YNP, only PARA-2 once co-occurred in the same sample together with GLOM-A13, which was found in six samples. As Paraglomus species form small, hyaline spores devoid of any prominent morphological characters, the possibility of cryptic species within this morphospecies appears plausible. Generally, the data available on the distribution of $P$. occultum and A. morrowiae also allow for the possibility that the occurrence of ACAU-6 and PARA-3 in geothermal soils can be attributed, in part, to their ability to tolerate low $\mathrm{pH}$ or the unique soil chemistry.

In many higher temperature samples, no phylotypes could be detected, resulting in the "NoSpecies" phylotype category strongly associated with temperature. This apparent absence of AMF was not due to PCR failure by inhibitor compounds as nontarget fungi were amplified from most of these samples. Samples were only taken from green, living plants and although microscopic examination revealed some degraded roots in the samples, intact roots devoid of AMF colonization were also present. We have observed that root mortality increases with soil temperature (unpublished data), which presents two possible explanations for the increased "NoSpecies" abundance in higher temperature soils: (1) plant roots may have a greater heat tolerance than AMF, or (2) 
decreased host quality by heat stress may cause lower colonization by the fungus. These two possibilities are currently being investigated under controlled greenhouse conditions using AMF isolates from YNP.

There is a complex relationship in the field between temperature and root growth. Soil temperatures in thermal areas increase with depth, and they fluctuate both diurnally and seasonally. The values for soil temperature were the temperature at the time we sampled and are, therefore, snapshots at a given time. In most field sites, there was a clear zonation from high-temperature areas where only $D$. lanuginosum grew, followed by areas with thermal $A$. scabra and $D$. lanuginosum, and a transition to more diverse meadow communities with nonthermal A. scabra. The latter grass could also be termed "thermal-influenced" due to the spatial proximity of the geothermal areas. In all sites, the difference between the average temperatures of thermal vs. nonthermal or control plants was at least $9.5^{\circ} \mathrm{C}$. Based on this, we believe we correctly identified ecologically relevant habitats in the field sites.

As thermal and nonthermal A. scabra may constitute different species [42], the possible effects of host preference on AMF communities cannot be separated from the influence of temperature in our study because different plant species occurred at specific temperature ranges. This is inevitable in a field study of this kind. Host preference is relatively low in the AM symbiosis but has been reported in the field $[13,44]$. In contrast to nonthermal A. scabra and $D$. lanuginosum, thermal $A$. scabra appears to be an annual [42]. This may explain the lower AMF richness in its roots because there is less time for the establishment of a complex AMF community. However, AMF taxa detected in thermal A. scabra generally represent a subset of those found in D. lanuginosum, and AMF in nonthermal $A$. scabra were more similar to control samples taken outside the geothermally influenced areas. Therefore, we consider the patterns we observed to be primarily due to habitat influence. In addition to temperature, Bunn and Zabinski [3] reported drastic differences in the soil chemical environment between thermal and adjacent thermally influenced soils. Unfortunately, soil chemical properties were not recorded in our samples and based on this, we cannot separate the effect of temperature from other variables that may change among the sample sites. This does not, however, influence our conclusion that the AMF communities appear to differ between different habitats.

The occurrence of distinct AMF in geothermal environmental soils does not provide evidence of a positive effect on the fitness of the plants by these fungi. Recently, a positive effect on Dichanthelium heat resistance by a different, unexpected group of fungi was reported, namely by ascomycete endophytes of the genus Curvularia [30]. This effect was attributed to a virus present in some of the
Curvularia strains [22]. We detected Curvularia as nontarget sequences in ten of our root samples (interestingly, not only in D. lanuginosum), but it was impossible to compare them to the Curvularia endophytes reported by Redman et al. [30] as the authors did not provide the respective sequences in the database. Nevertheless, these findings suggest the possibility that a wide range of symbiotic interactions may be involved in heat tolerance of plants in YNP.

In summary, we detected the presence of both generalist and potential specialist AMF in geothermal areas of YNP. The communities observed appeared to be different from those located in nonthermal areas, but whether or not this was caused by temperature or other edaphic factors remains uncertain.

Acknowledgments This work was funded by a grant from the Thermal Biology Institute, Montana State University, to C.Z and D.R., which is gratefully acknowledged. The authors also would like to thank the following persons: Thomas Boller and Andres Wiemken at the Botanical Institute, University of Basel for continuing support of D.R.; Rebecca Bunn, Tracy McCreery, and Lorna McIntyre for their help in taking samples in Yellowstone; Zuzana Sýkorová and Isabelle Hijri for sequencing; Thomas Wohlgemuth for help with multivariate analysis; Hafdis Hanna Aegisdottir and Ulfur Oskarsson for help in finding field sites and sampling in Iceland; Christie Hendrix (Yellowstone National Park Research Office) for administrative help.

\section{References}

1. Altschul SF, Madden TL, Schaeffer AA, Zhang J, Zhang Z, Miller W, Lipman DJ (1997) Gapped BLAST and PSI-BLAST: A new generation of protein database search programs. Nuc Ac Res 25:3389-3402

2. Borowicz V (2001) Do arbuscular mycorrhizal fungi alter plantpathogen relations. Ecology 82:3057-3068

3. Bunn RA, Zabinski CA (2003) Arbuscular mycorrhizae in thermal-influenced soils in Yellowstone National Park. West $\mathrm{N}$ Am Naturalist 63:409-415

4. Clark RB (1997) Arbuscular mycorrhizal adaptation, spore germination, root colonization, and host plant growth and mineral acquisition at low pH. Plant Soil 192:15-22

5. Clark RB, Zobel RW, Zeto SK (1999) Effects of mycorrhizal fungus isolates on mineral acquisition by Panicum virgatum in acidic soil. Mycorrhiza 9:167-176

6. Colwell RC (2005) EstimateS: Statistical estimation of species richness and shared species from samples. Version 7.5. Accessed at http://purl.oclc.org/estimates

7. Dickie IA, Avis PG, McLaughlin DJ, Reich PB (2003) GoodEnough RFLP Matcher (GERM) program. Mycorrhiza 13:171172

8. Fitter AH (2005) Darkness visible: reflections on underground ecology. J Ecol 93:231-243

9. Gavito M, Olsson P, Rouhier H, Medina-Penafiel A, Jakobsen I, Bago A, Azcon-Aguilar C (2005) Temperature constraints on the growth and functioning of root organ cultures with arbuscular mycorrhizal fungi. New Phytol 168:179-188

10. George HL, Davies FS, Crane JH, Schaffer B (2002) Root temperature effects on 'Arkin' carambola (Averrhoa carambola L.) trees II. Growth and mineral nutrition. Sci Horticult Amsterdam 96:67-79 
11. Germino MJ, Wraith JM (2003) Plant water relations influence carbon gain in a grass occurring along sharp gradients of soil temperature. New Phytol 157:241-250

12. Heinemeyer A, Ridgway KP, Edwards EJ, Benham DG, Young JPW, Fitter AH (2004) Impact of soil warming and shading on colonization and community structure of arbuscular mycorrhizal fungi in roots of a native grassland community. Glob Chang Biol 10:52-64

13. Helgason T, Merryweather JW, Denison J, Wilson P, Young JPW, Fitter AH (2002) Selectivity and functional diversity in arbuscular mycorrhizas of co-occurring fungi and plants from a temperate deciduous woodland. J Ecol 90:371-384

14. Henley RW, Hedenquist JW, Roberts PJ (1986) Guide to the active epithermal (geothermal) systems and precious metal deposits of New Zealand. Monograph Series on Mineral Deposits. Gebrüder Borntraeger, Berlin

15. Hijri I, Sýkorová Z, Oehl F, Ineichen K, Mäder P, Wiemken A, Redecker D (2006) Communities of arbuscular mycorrhizal fungi in arable soils are not necessarily low in diversity. Mol Ecol 15:2277-2289

16. Husband R, Herre EA, Turner SL, Gallery R, Young JPW (2002) Molecular diversity of arbuscular mycorrhizal fungi and patterns of host association over time and space in a tropical forest. Mol Ecol 11:2669-2678

17. Jansa J, Mozafar A, Banke S, McDonald BA, Frossard E (2002) Intra- and intersporal diversity of ITS rDNA sequences in Glomus intraradices assessed by cloning and sequencing, and by SSCP analysis. Mycol Res 106:670-681

18. Koch A, Kuhn G, Fontanillas P, Fumagalli L, Goudet J, Sanders IR (2004) High genetic variability and low local diversity in a population of arbuscular mycorrhizal fungi. Proc Natl Acad Sci U S A 101:2369-2374

19. Koske RE, Gemma JN (1989) A modified procedure for staining roots to detect VA mycorrhizas. Mycol Res 92:486-505

20. Kvist T, Ahring BK, Westermann P (2007) Archaeal diversity in Icelandic hot springs. FEMS Microbiol Ecol 59:71-80

21. Lekberg Y, Koide RT, Rohr JR, Aldrich-Wolfe L, Morton JB (2007) Role of niche restrictions and dispersal in the composition of arbuscular mycorrhizal fungal communities. J Ecol 95:95-105

22. Marquez LM, Redman RS, Rodriguez RJ, Roossinck MJ (2007) A virus in a fungus in a plant: Three-way symbiosis required for thermal tolerance. Science 315:513-515

23. Morton JB, Walker C (1984) Glomus diaphanum — a new species in the Endogonaceae common in West Virginia. Mycotaxon $21: 431-440$

24. Morton JB, Redecker D (2001) Two new families of Glomales, Archaeosporaceae and Paraglomaceae, with two new genera Archaeospora and Paraglomus, based on concordant molecular and morphological characters. Mycologia 93:181-195

25. Öpik M, Moora M, Liira J, Zobel M (2006) Composition of rootcolonizing arbuscular mycorrhizal fungal communities in different ecosystems around the globe. J Ecol 94:778-790

26. Posada D (2004) Modeltest 3.5. Facultad de Biologia, Universidad de Vigo, Vigo

27. Redecker D (2002) Molecular identification and phylogeny of arbuscular mycorrhizal fungi. Plant Soil 244:67-73

28. Redecker D, Hijri M, Dulieu H, Sanders IR (1999) Phylogenetic analysis of a dataset of fungal 5.8S rDNA sequences shows that highly divergent copies of Internal Transcribed Spacers reported from Scutellospora castanea are of Ascomycete origin. Fung Genet Biol 28:238-244
29. Redecker D, Morton JB, Bruns TD (2000) Ancestral lineages of arbuscular mycorrhizal fungi (Glomales). Mol Phylogen Evol $14: 276-284$

30. Redman RS, Sheehan KB, Stout RG, Rodriguez RJ, Henson JM (2002) Thermotolerance generated by plant/fungal symbiosis. Science 298:1581

31. Renker C, Blaszkowski J, Buscot F (2007) Paraglomus laccatum comb. nov-a new member of Paraglomeraceae (Glomeromycota). Nova Hedwig 84:395-407

32. Rodman A, Shovic H, Thoma D (1996) Soils of Yellowstone National Park. Yellowstone Center for Resources YCR-NRSR-962, National Park Service, Yellowstone National Park

33. Russell AJ, Bidartondo MI, Butterfield BG (2002) The root nodules of the Podocarpaceae harbour arbuscular mycorrhizal fungi. New Phytol 156:283-295

34. Schwarzott D, Walker C, Schüßler A (2001) Glomus, the largest genus of the arbuscular mycorrhizal fungi (Glomales), is nonmonophyletic. Mol Phylogen Evol 21:190-197

35. Smith SE, Read DJ (1997) Mycorrhizal symbiosis, 2nd edn. Academic, London

36. Spain JL, Sieverding E, Oehl F (2006) Ambispora: a new genus in the arbuscular mycorrhiza-forming Glomeromycetes, with a discussion of the genus Archaeospora. Mycotaxon 97:163182

37. Stout RG, Summers ML, Kerstetter T, McDermott TR (1997) Heat- and acid-tolerance of a grass commonly found in geothermal areas within Yellowstone National Park. Plant Sci 130:1-9

38. Stout RG, Al-Niemi TS (2002) Heat-tolerant flowering plants of active geothermal areas in Yellowstone National Park. Ann Bot 90:259-267

39. Swofford DL (2001) PAUP*. Phylogenetic Analysis Using Parsimony (*and Other Methods). Sinauer, Sunderland

40. Sýkorová Z, Wiemken A, Redecker D (2007) Co-occurring Gentiana verna and Gentiana acaulis and their neighboring plants in two Swiss upper montane meadows harbor distinct arbuscular mycorrhizal fungal communities. App Environ Microbiol 73:5426-5434

41. ter Braak CJF, Smilauer P (2004) CANOCO reference manual and CanoDraw for Windows user's guide: software for canonical community ordination (version 4.5). Biometris, Wageningen

42. Tercek MT, Whitbeck JL (2004) Heat avoidance life history strategy controls the distribution of geothermal Agrostis in yellowstone. Ecology 85:1955-1966

43. Tercek MT, Hauber DP, Darwin SP (2003) Genetic and historical relationships among geothermally adapted Agrostis (bentgrass) of North America and Kamchatka: evidence for a previously unrecognized, thermally adapted taxon. Am J Bot 90:13061312

44. Vandenkoornhuyse P, Ridgway KP, Watson IJ, Fitter AH, Young JPW (2003) Co-existing grass species have distinctive arbuscular mycorrhizal communities. Mol Ecol 12:3085-3095

45. Walker C (2008) Mycological research news: Ambispora and Ambisporaceae resurrected. Mycol Res 112:297-298

46. White TJ, Bruns T, Lee S, Taylor J (1990) Amplification and direct sequencing of fungal ribosomal RNA genes for phylogenetics. In: Innis MA et al (ed) PCR protocols, a guide to methods and applications. Academic, London, pp 315-322

47. Wubet T, Weiss M, Kottke I, Teketay D, Oberwinkler F (2004) Molecular diversity of arbuscular mycorrhizal fungi in Prunus africana, an endangered medicinal tree species in dry Afromontane forests of Ethiopia. New Phytol 161:517-528 